typeset using JPSJ.sty $<$ ver.1.0b $>$

\title{
Application of the Cluster Variation Method to Spin Ice Systems on the Pyrochlore Lattice
}

\author{
Shun-ichi Yoshida*, Koji Nemoto, Koh WAdA \\ Division of Physics, Graduate School of Science, Hokkaido University, Sapporo 060-0810
}

(Received November 2, 2018)

\begin{abstract}
The cactus approximation in the cluster variation method is applied to the spin ice system with nearest neighbor ferromagnetic coupling. The temperature dependences of the entropy and the specific heat show qualitatively good agreement with those observed by Monte Carlo simulations and experiments, and the Pauling value is reproduced for the residual entropy. The analytic expression of the $\boldsymbol{q}$-dependent magnetic susceptibility is obtained, from which the absence of magnetic phase transition is confirmed. The neutron scattering pattern is also evaluated and found to be consistent with that obtained from Monte Carlo simulations.
\end{abstract}

KEYWORDS: Spin Ice, Geometrical Frustration System, Cluster Variation Method, Cactus Approximation.

\section{§1. Introduction}

In recent years the low temperature physics of 'spin ice' systems such as $\mathrm{Ho}_{2} \mathrm{Ti}_{2} \mathrm{O}_{3}$ and $\mathrm{Dy}_{2} \mathrm{Ti}_{2} \mathrm{O}_{3}$ draws a great deal of attentions of many researchers. Among them the most remarkable property is that such materials have a residual entropy whose value is almost the same as that obtained by Pauling ${ }^{1)}$ for proton configurations in the ordinary hexagonal ice $\mathrm{I}_{\mathrm{h}}$. This indicates the existence of the 'ice rule' in these magnetic materials ${ }^{2)}$ due to spin frustrations. The difference of 'spin ice' from the real ice is that we can resolve the degeneracy by applying an infinitesimal magnetic field. One of our interests then is how the spins behave in response to magnetic fields. The spin ice system has a spin lattice forming the pyrochlore structure as depicted in Fig. 1(a). In the pyrochlore lattice the spin sites construct a three dimensional network of corner-sharing tetrahedra with cubic symmetry. Each site belongs to two tetrahedra and the spin is forced to point toward either of these centers by strong crystal field anisotropy. Due to this Ising-like property the spin system becomes fully-frustrated on the pyrochrore lattice if the neighboring spins are likely to align ferromagnetically because the direction-cosine between their easy-axes is negative. In this case the lowest energy of four-spin state on a tetrahedron is given by the configurations where two spins point "in" and the other two spins "out", and it is six-fold degenerated (see Fig. 1(c)). This rule applied in the ground state is the same as that for the proton configuration in the ordinary hexagonal ice $\mathrm{I}_{\mathrm{h}}$, and is therefore called the ice rule. The ice rule makes the number of low-lying states of the whole system enormous as long as the long range spin-spin interactions are very weak. In particular the ground state is macroscopically degenerated when only the nearest neighbor interaction is concerned. Pauling is the first one who

\footnotetext{
* E-mail: shun1@statphys.sci.hokudai.ac.jp
}

estimated the value of the residual entropy to obtain

$$
\frac{S_{0}}{N k_{\mathrm{B}}}=\frac{1}{2} \ln \frac{3}{2} \simeq 0.203
$$

where $N$ is the number of protons in the ice $\mathrm{I}_{\mathrm{h}}$. Although his original interest was the entropy due to the proton configurations in the ordinary hexagonal ice $\mathrm{I}_{\mathrm{h}}$, the value obtained is the same for the pyrochlore lattice since the network topology of tetrahedra is ignored in his estimation. It turned out that the difference between the structure of tetrahedra in the pyrochlore and $\mathrm{I}_{\mathrm{h}}$ lattices is not so relevant because the correction to the Pauling value is very small. ${ }^{3)}$ This is the reason why the Pauling value is a good approximate one for both systems. Indeed the specific heat for spin ice systems was measured by experiments and by Monte Carlo simulations, ${ }^{4)}$ from which the residual entropy is evaluated and agrees with (1.1). A mean field approximation (MFA) may be the first step to examine thermodynamic properties and has been so far applied to the spin ice system to analyze neutron scattering experiments. ${ }^{5) 6)}$ The simplest MFA is the one using the 4-sublattice magnetizations and neglecting spin correlations. Such a treatment, however, results in a phase transition at a finite temperature, below which a long range order appears and brings about the vanishing of the entropy as $T \rightarrow 0 .{ }^{5)}$ This discrepancy from those of experiments and Monte Carlo simulation is due to the fact that the MFA cannot deal with the spin fluctuations and "two-in and two-out" spin behaviors in tetrahedra, i.e., the ice rule.

On the other hand, Slater studied a hydrogen bonded crystal $\mathrm{KH}_{2} \mathrm{PO}_{4}(\mathrm{KDP})^{7)}$ with the ice rule. In this crystal the ice rule is described by that (i) one proton exists on each $\mathrm{O}-\mathrm{O}$ bond between two nearest neighboring $\mathrm{PO}_{4}$ tetrahedra and (ii) each tetrahedron has exactly two protons ajacent to it. He succeded in explaining the phase transition of KDP by coping with the ice rule. His approximate treatment is tantamount to an extended Bethe approximation. It is equivalent to the tetrahedral cactus approximation in the cluster variation method (CVM), 
which was developed to improve the MFA systematically by taking into account higher order correlations. ${ }^{8)}$ The tetrahedral cactus approximation in the CVM was successfully applied to analyze the wave-number dependent ferro-electric susceptibility above and below the transition temperature for the KDP crystal. ${ }^{9)}$

In the present paper, we apply the tetrahedral cactus approximation in the CVM to the spin ice system with nearest-neighbor ferromagnetic coupling. Within the present approximation we show that no magnetic phase transition takes place by investigating analytically obtained eigenvalues appearing in the wave-number dependent susceptibility. As a result the Pauling value for the residual entropy is reproduced in the zero temperature limit. Furthermore we evaluate the neutron scattering intensity, which is qualitatively in good agreement with that obtained by Monte Carlo simulations and experiments.

The present manuscript is organized as follows: In $\S 2$ we present the CVM formulation for the spin ice system with nearest neighbor ferromagnetic coupling to derive a set of linear response equations to an inhomogeneous magnetic field. In $\S 3$ described are the main results including the temperature dependency of the entropy and of the specific heat, the wave-number dependent susceptibility and the neutron scattering pattern. Section 4 is devoted to a summary of this manuscript with some discussions on the relation between the present approximation and the usual mean field approximation.

\section{$\S 2$. Formulation of Nearest Neighbor Spin Ice System}

\subsection{Indexing Site Locations}

The pyrochlore lattice is a non-Bravias lattice. In a rhombohedral chemical unit cell located at a translational lattice site $\boldsymbol{R}$ there exist four independent spins situated on a tetrahedron whose position vectors are denoted by $\boldsymbol{r}_{\nu}$ relative to $\boldsymbol{R}$. Thus there are four sublattices for spin configurations in the pyrochlore lattice. For $N / 4$ rhombohedral chemical unit cells the translational lattice vector $\boldsymbol{R}$ is chosen in the center of each unit cell. The $\nu$-th position vector in the unit cell is taken, respectively, as

$$
\begin{aligned}
& \boldsymbol{r}_{1}=\frac{a}{4}\left(\begin{array}{l}
0 \\
0 \\
0
\end{array}\right), \quad \boldsymbol{r}_{2}=\frac{a}{4}\left(\begin{array}{l}
0 \\
1 \\
1
\end{array}\right), \\
& \boldsymbol{r}_{3}=\frac{a}{4}\left(\begin{array}{l}
1 \\
0 \\
1
\end{array}\right), \quad \boldsymbol{r}_{4}=\frac{a}{4}\left(\begin{array}{l}
1 \\
1 \\
0
\end{array}\right),
\end{aligned}
$$

where $a$ is the linear size of the cubic primitive cell (see Fig.1(a)). For later notational convenience we sometimes use the sequential numbering $i$-th site $(i=1, \cdots, N)$ for a spin site $\boldsymbol{R}+\boldsymbol{r}_{\nu}$.

\subsection{Spin Hamiltonian}

The Hamiltonian of the spin ice system with $N$ spins under an inhomogeneous external field $\boldsymbol{H}_{i}(i=$ (a)

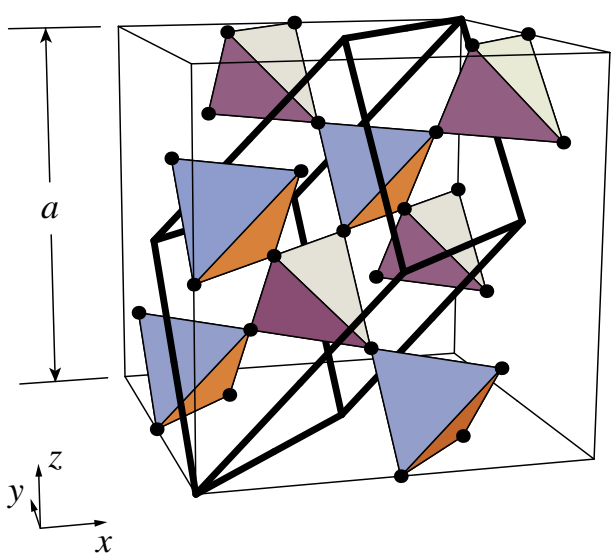

(b)

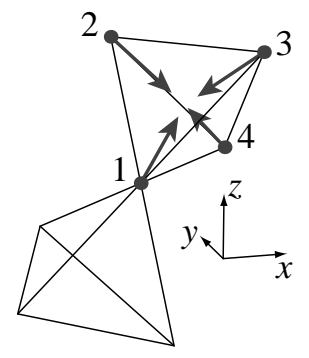

(c)

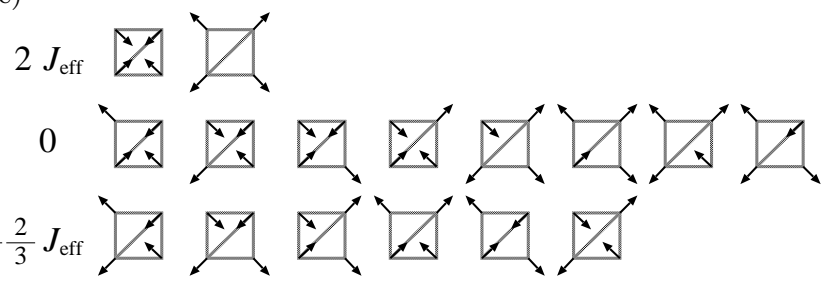

Fig. 1. (a) A schematic plot of the pyrochlore lattice. The magnetic ions are located at each vertex of the tetrahedra. The outlines of the rhombohedral chemical unit-cell and the cubic unit-cell are also shown. There are four independent spins and two tetrahedra in a rhombohedral chemical unit-cell. (b) Local Ising axes $\boldsymbol{n}_{\nu}(\nu=1,2,3,4)$ (c) Energy levels of spin configurations in one tetrahedron.

$1, \cdots, N)$ is given as

$$
\begin{aligned}
& \mathcal{H}\left(\boldsymbol{S}_{1}, \cdots, \boldsymbol{S}_{N}\right) \\
& =-D_{\mathrm{a}} \sum_{i}\left(\boldsymbol{n}_{\nu(i)} \cdot \boldsymbol{S}_{i}\right)^{2}-J \sum_{\langle i j\rangle} \boldsymbol{S}_{i} \cdot \boldsymbol{S}_{j}-\sum_{i} \boldsymbol{H}_{i} \cdot \boldsymbol{S}_{i} \\
& \quad+D r_{\mathrm{nn}}^{3} \sum_{\langle i j\rangle}\left[\frac{\boldsymbol{S}_{i} \cdot \boldsymbol{S}_{j}}{\left|\boldsymbol{r}_{i j}\right|^{3}}-\frac{3\left(\boldsymbol{S}_{i} \cdot \boldsymbol{r}_{i j}\right)\left(\boldsymbol{S}_{j} \cdot \boldsymbol{r}_{i j}\right)}{\left|\boldsymbol{r}_{i j}\right|^{5}}\right],
\end{aligned}
$$

where $\boldsymbol{S}_{i}$ is a spin vector at the $i$-th site $\left(\left|\boldsymbol{S}_{i}\right|=1\right), \boldsymbol{r}_{i j}$ the displacement vector from the $j$-th to the $i$-th site, and $r_{\mathrm{nn}}$ is the distance between nearest neighboring two spins. The second term represents the nearest-neighbor ferromagnetic exchange interactions $(J>0)$. The third and the last terms are the Zeeman energy and the dipolar interactions, respectively. The first term, which repre- 
sents the single-ion anisotropy $\left(D_{\mathrm{a}}>0\right)$, is the most crucial one in the spin ice system because this term makes the system obey the ice rule. There are four distinct local easy-axis directions denoted by $\boldsymbol{n}_{\nu(i)}$, where $\nu$ denotes the sublattice where the $i$-th spin belongs. As shown in Fig. 1(b) we take the unit vector $\boldsymbol{n}_{\nu}$ directing the local Ising axis of the $\nu$-th sublattice as

$$
\begin{aligned}
& \boldsymbol{n}_{1}=\frac{1}{\sqrt{3}}\left(\begin{array}{l}
1 \\
1 \\
1
\end{array}\right), \quad \boldsymbol{n}_{2}=\frac{1}{\sqrt{3}}\left(\begin{array}{r}
1 \\
-1 \\
-1
\end{array}\right), \\
& \boldsymbol{n}_{3}=\frac{1}{\sqrt{3}}\left(\begin{array}{r}
-1 \\
1 \\
-1
\end{array}\right), \quad \boldsymbol{n}_{4}=\frac{1}{\sqrt{3}}\left(\begin{array}{r}
-1 \\
-1 \\
1
\end{array}\right) .
\end{aligned}
$$

Now we introduce two assumptions to make the model a little simpler. First we consider the case where the dipole interactions are so weak $(D \ll J)$ that they are negligible except the nearest-neighbor one, although one has to cope with the effect of $D$ when expecting that the system falls into the unique ground state in very low temperatures. Second the single-ion anisotropy is so strong $\left(D_{\mathrm{a}} \gg J\right)$ that the spins are forced to align along their uniaxial directions. Then each of the spins $\boldsymbol{S}_{i}$ can be expressed with an Ising variable $\sigma_{i}$ as

$$
\boldsymbol{S}_{i}=\sigma_{i} \boldsymbol{n}_{\nu(i)}, \quad \sigma_{i}= \pm 1
$$

and the Hamiltonian (2.2) can be expressed in terms of $\left\{\sigma_{i}\right\}$ as

$$
\mathcal{H}\left(\sigma_{1}, \cdots, \sigma_{N}\right) \simeq \frac{J_{\mathrm{eff}}}{3} \sum_{\langle i j\rangle} \sigma_{i} \sigma_{j}-\sum_{i} H_{i} \sigma_{i},
$$

where $J_{\text {eff }}=J-5 D(>0)$ and $H_{i}=\boldsymbol{H}_{i} \cdot \boldsymbol{n}_{\nu(i)}$. Note that the interaction term is antiferromagnetic, which means that the system is fully-frustrated.

For the convenience of the following approximation, it is useful to divide the above Hamiltonian into those for the tetrahedra:

$$
\mathcal{H}\left(\sigma_{1}, \cdots, \sigma_{N}\right)=\sum_{\langle i j k l\rangle} \mathcal{H}_{4}^{(i j k l)}\left(\sigma_{i}, \sigma_{j}, \sigma_{k}, \sigma_{l}\right),
$$

where the superscript $(i j k l)$ denotes a set of four spin sites on a tetrahedron and the index $\langle i j k l\rangle$ runs over all tetrahedra. The Hamiltonian in a tetrahedron $\mathcal{H}_{4}^{(i j k l)}$ consists of the interaction term $\mathcal{H}_{4}^{0}$ and the Zeeman term $\Delta \mathcal{H}_{4}^{(i j k l)}$ :

$$
\begin{aligned}
& \mathcal{H}_{4}^{(i j k l)}\left(\sigma_{i}, \sigma_{j}, \sigma_{k}, \sigma_{l}\right) \\
& =\mathcal{H}_{4}^{0}\left(\sigma_{i}, \sigma_{j}, \sigma_{k}, \sigma_{l}\right)+\Delta \mathcal{H}_{4}^{(i j k l)}\left(\sigma_{i}, \sigma_{j}, \sigma_{k}, \sigma_{l}\right),
\end{aligned}
$$

where

$$
\begin{aligned}
\mathcal{H}_{4}^{0} & \left(\sigma_{i}, \sigma_{j}, \sigma_{k}, \sigma_{l}\right) \\
& =\frac{J_{\mathrm{eff}}}{3}\left(\sigma_{i} \sigma_{j}+\sigma_{j} \sigma_{k}+\sigma_{k} \sigma_{l}+\sigma_{l} \sigma_{i}+\sigma_{i} \sigma_{k}+\sigma_{j} \sigma_{l}\right),
\end{aligned}
$$

$$
\begin{aligned}
& \Delta \mathcal{H}_{4}^{(i j k l)}\left(\sigma_{i}, \sigma_{j}, \sigma_{k}, \sigma_{l}\right) \\
& \quad=-\frac{1}{2}\left(H_{i} \sigma_{i}+H_{j} \sigma_{j}+H_{k} \sigma_{k}+H_{l} \sigma_{l}\right) .
\end{aligned}
$$

\subsection{Cactus Approximation in the CVM}

The starting point of the cluster variation method (CVM) is the following well-known variation principle. Let $P_{\text {tot }}\left(\sigma_{1}, \cdots, \sigma_{N}\right)$ be the probability of a whole spin configuration. Then the variational free energy of the system $F$ is given by

$$
\begin{aligned}
\beta F=\operatorname{Tr} & {\left[\beta \mathcal{H}\left(\sigma_{1}, \cdots, \sigma_{N}\right) P_{\text {tot }}\left(\sigma_{1}, \cdots, \sigma_{N}\right)\right] } \\
& +\operatorname{Tr}\left[P_{\text {tot }}\left(\sigma_{1}, \cdots, \sigma_{N}\right) \ln P_{\text {tot }}\left(\sigma_{1}, \cdots, \sigma_{N}\right)\right],
\end{aligned}
$$

where $\beta=1 / k_{\mathrm{B}} T$. The equilibrium probability is obtained by minimizing $F$ with respect to $P_{\text {tot }}\left(\sigma_{1}, \cdots, \sigma_{N}\right)$. Then systematic approximations to $P_{\text {tot }}$ correspond to those to the free energy one by one. For example the 'point' approximation

$$
P_{\mathrm{tot}}\left(\sigma_{1}, \cdots, \sigma_{N}\right) \simeq \prod_{i} P_{1}^{(i)}\left(\sigma_{i}\right),
$$

corresponds to the usual mean field approximation where $P_{1}^{(i)}\left(\sigma_{i}\right)$ denotes the single spin probability. In the present work we adopt the tetrahedral 'cactus' approximation, whose approximant is written as

$$
\begin{aligned}
& P_{\text {tot }}\left(\sigma_{1}, \cdots, \sigma_{N}\right) \\
& \simeq \prod_{i} P_{1}^{(i)}\left(\sigma_{i}\right) \prod_{\langle i j k l\rangle} \frac{P_{4}^{(i j k l)}\left(\sigma_{i}, \sigma_{j}, \sigma_{k}, \sigma_{l}\right)}{P_{1}^{(i)}\left(\sigma_{i}\right) P_{1}^{(j)}\left(\sigma_{j}\right) P_{1}^{(k)}\left(\sigma_{k}\right) P_{1}^{(l)}\left(\sigma_{l}\right)},
\end{aligned}
$$

where the probability for the four spins $P_{4}^{(i j k l)}\left(\sigma_{i}, \sigma_{j}, \sigma_{k}, \sigma_{l}\right)$ is introduced to take into account two spins 'in' and two spins 'out' correlation on a tetrahedron. Substituting (2.12) into (2.10) with (2.6) we obtain the following expression for the corresponding variational free energy:

$$
\begin{aligned}
\beta F \simeq & \sum_{\langle i j k l\rangle} \operatorname{Tr}\left[\beta \mathcal{H}_{4}^{(i j k l)}\left(\sigma_{i}, \sigma_{j}, \sigma_{k}, \sigma_{l}\right)\right. \\
& \left.\times P_{4}^{(i j k l)}\left(\sigma_{i}, \sigma_{j}, \sigma_{k}, \sigma_{l}\right)\right] \\
& -\sum_{i} \operatorname{Tr}\left[P_{1}^{(i)}\left(\sigma_{i}\right) \ln P_{1}^{(i)}\left(\sigma_{i}\right)\right] \\
& +\sum_{\langle i j k l\rangle} \operatorname{Tr}\left[P_{4}^{(i j k l)}\left(\sigma_{i}, \sigma_{j}, \sigma_{k}, \sigma_{l}\right)\right. \\
& \left.\times \ln P_{4}^{(i j k l)}\left(\sigma_{i}, \sigma_{j}, \sigma_{k}, \sigma_{l}\right)\right] .
\end{aligned}
$$

Since there are normalization conditions for $P_{1}$ and $P_{4}$, these probability functions are not always independent variational parameters to each other. To eliminate these restrictions we define order parameters as a set of independent variables,

$$
\begin{aligned}
m_{a} & =\operatorname{Tr}\left[\sigma_{a} P_{4}^{(i j k l)}\left(\sigma_{i}, \sigma_{j}, \sigma_{k}, \sigma_{l}\right)\right], \\
m_{a b} & =\operatorname{Tr}\left[\sigma_{a} \sigma_{b} P_{4}^{(i j k l)}\left(\sigma_{i}, \sigma_{j}, \sigma_{k}, \sigma_{l}\right)\right], \\
m_{a b c} & =\operatorname{Tr}\left[\sigma_{a} \sigma_{b} \sigma_{c} P_{4}^{(i j k l)}\left(\sigma_{i}, \sigma_{j}, \sigma_{k}, \sigma_{l}\right)\right], \\
m_{i j k l} & =\operatorname{Tr}\left[\sigma_{i} \sigma_{j} \sigma_{k} \sigma_{l} P_{4}^{(i j k l)}\left(\sigma_{i}, \sigma_{j}, \sigma_{k}, \sigma_{l}\right)\right] .
\end{aligned}
$$

where $m_{a}$ and $m_{a b c}$ represent long-range order parameters and $m_{a b}$ and $m_{i j k l}$ short-range order parameters, respectively, and $a, b, c \in\{i, j, k, l\}$. In terms of these order parameters we can express the probabilities $P_{1}$ and $P_{4}$ as

$$
P_{1}^{(i)}\left(\sigma_{i}\right)=\frac{1}{2}\left(1+m_{i} \sigma_{i}\right)
$$




$$
\begin{aligned}
& P_{4}^{(i j k l)}\left(\sigma_{i}, \sigma_{j}, \sigma_{k}, \sigma_{l}\right) \\
& =\frac{1}{2^{4}}\left(1+\sum_{a} m_{a} \sigma_{a}+\sum_{a b} m_{a b} \sigma_{a} \sigma_{b}\right. \\
& \left.\quad+\sum_{a b c} m_{a b c} \sigma_{a} \sigma_{b} \sigma_{c}+m_{i j k l} \sigma_{i} \sigma_{j} \sigma_{k} \sigma_{l}\right)
\end{aligned}
$$

Now that the variational free energy is expressed in terms of the order parameters, the thermal equilibrium conditions, in other words, the equations of state can be given by its extremum condition:

$\frac{\partial F}{\partial m_{a}}=0, \quad \frac{\partial F}{\partial m_{a b}}=0, \quad \frac{\partial F}{\partial m_{a b c}}=0, \quad \frac{\partial F}{\partial m_{i j k l}}=0$.

\section{§3. Results from Cactus Approximation}

\subsection{Entropy and Specific Heat}

In the case of no external field $\left(H_{i}=0\right)$ we can derive the analytic expression for the probabilities $P_{1}$ and $P_{4}$ as

$$
\begin{aligned}
& P_{1}^{0}(\sigma)=1 / 2, \\
& P_{4}^{0}\left(\sigma_{i}, \sigma_{j}, \sigma_{k}, \sigma_{l}\right)=\mathrm{e}^{-\beta \mathcal{H}_{4}^{0}\left(\sigma_{i}, \sigma_{j}, \sigma_{k}, \sigma_{l}\right)} / Z, \\
& Z=\operatorname{Tr}\left[\mathrm{e}^{-\beta \mathcal{H}_{4}^{0}\left(\sigma_{i}, \sigma_{j}, \sigma_{k}, \sigma_{l}\right)}\right]=6 \eta^{-1}+8+2 \eta^{3},
\end{aligned}
$$

where $\eta=\exp \left(-2 J_{\text {eff }} / 3 k_{\mathrm{B}} T\right)$ and the superscript " 0 " is used to emphasize thermodynamical equilibrium without external field. In deriving the above solution we assumed that no long range order state happens at finite temperature, which is indeed the case as we will see later. It is straightforward to calculate the entropy $S$ and the specific heat $C=T(\mathrm{~d} S / \mathrm{d} T)$ by substituting the above equations in the variational free energy (2.13) to obtain

$$
\frac{S}{N k_{\mathrm{B}}}=-\ln 2+\frac{2 J_{\mathrm{eff}}\left(\eta^{3}-\eta^{-1}\right)}{k_{\mathrm{B}} T Z}+\frac{1}{2} \ln Z,
$$

The temperature dependence of $S$ and $C$ are shown in Fig. 2. We see that the entropy $S$ takes the Pauling value (1.1) in the limit $T \rightarrow 0$ and the specfic heat $C$ shows a Schottky anomaly as is expected. These behaviors are in agreement with those observed in experiments and in Monte Carlo simulations. ${ }^{4}$ ) For comparison the results obtained from the mean field approximation (MFA) are also shown by thin curves. Note that the long range order makes both $S$ and $C$ look very different in the low temperature region including $T=0$.

\subsection{Wave-Number dependent Susceptibility}

Here we examine the magnetic property by using the linear response to an inhomogeneous magnetic field $H_{i}$, in which eqs.(3.1)-(3.3) become the bases of linearization. Some calculations lead us to the linear equations between magnetization $\Delta m_{i}\left(=m_{i}\right)$ and magnetic field. The detailed derivation is described in appendix $\mathrm{A}$ and the linear response equation is given by

$$
\beta H\left(\boldsymbol{R}+\boldsymbol{r}_{\nu}\right)=\sum_{\nu^{\prime}, \boldsymbol{R}^{\prime}} \Lambda_{\nu \nu^{\prime}} \Delta m\left(\boldsymbol{R}^{\prime}+\boldsymbol{r}_{\nu^{\prime}}\right),
$$

where we use the indexing rule described in $\S 2.1$ to express the site location. The index $\left(\nu^{\prime}, \boldsymbol{R}^{\prime}\right)$ runs over all nearest neighbors of the site $(\nu, \boldsymbol{R})$. The elements of $4 \times 4$
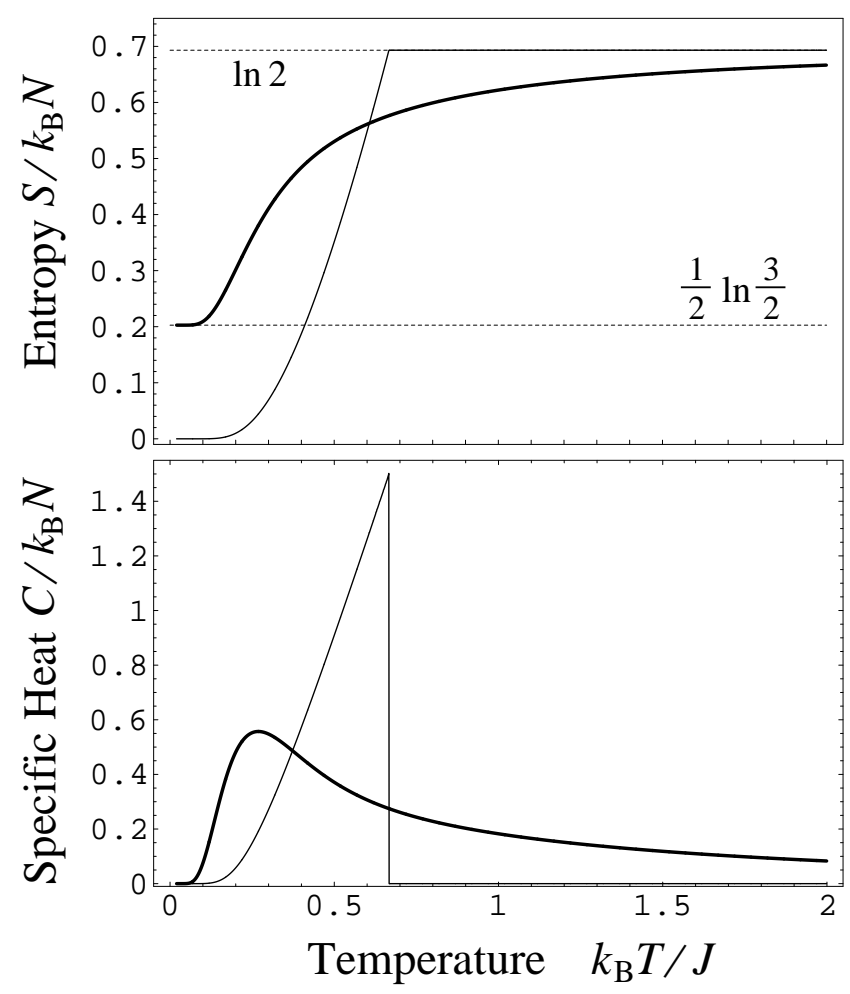

Fig. 2. Entropy and specific heat versus temperature. Entropy at $T=0$ agrees with the Pauling value (1.1). Specific heat shows the Schottky anomaly. Thin curves are results obtained from the mean field approximation.

matrix $\Lambda$ are given as

$$
\begin{aligned}
& \Lambda_{\nu \nu^{\prime}}=\Lambda_{\text {off }}+\left(\Lambda_{\text {diag }}-\Lambda_{\text {off }}\right) \delta_{\nu \nu^{\prime}} \\
& \Lambda_{\text {diag }}=-\frac{1}{2}+\frac{Z d_{1}}{16}, \quad \Lambda_{\text {off }}=\frac{Z d_{2}}{16}
\end{aligned}
$$

with

$$
d_{1}=\frac{1+3 \eta-3 \eta^{2}+3 \eta^{3}}{2\left(1+\eta^{3}\right)}, \quad d_{2}=\frac{1-\eta+\eta^{2}-\eta^{3}}{2\left(1+\eta^{3}\right)}
$$

In order to obtain the magnetic susceptibility from eq.(3.5) we decompose it into the Fourier components by utilizing the translational vector $\boldsymbol{R}$. We define the Fourier transformation of $\Delta m, H, \Lambda_{\nu \nu^{\prime}}$ for each sublattice as

$$
\begin{aligned}
& \Delta m_{\boldsymbol{q}_{;} \nu}=\mathrm{e}^{-\mathrm{i} \boldsymbol{q} \cdot \boldsymbol{r}_{\nu}} \sum_{\boldsymbol{R}} \mathrm{e}^{-\mathrm{i} \boldsymbol{q} \cdot \boldsymbol{R}} \Delta m\left(\boldsymbol{R}+\boldsymbol{r}_{\nu}\right), \\
& H_{\boldsymbol{q}_{; \nu}}=\mathrm{e}^{-\mathrm{i} \boldsymbol{q} \cdot \boldsymbol{r}_{\nu}} \sum_{\boldsymbol{R}} \mathrm{e}^{-\mathrm{i} \boldsymbol{q} \cdot \boldsymbol{R}} H\left(\boldsymbol{R}+\boldsymbol{r}_{\nu}\right) \\
& \Lambda_{\boldsymbol{q} ; \nu \nu^{\prime}}=2 \Lambda_{\nu \nu^{\prime}} \cos \left(\boldsymbol{q} \cdot\left(\boldsymbol{r}_{\nu}-\boldsymbol{r}_{\nu^{\prime}}\right)\right) .
\end{aligned}
$$

Then eq.(3.5) is reduced to the linear relation for sublattice magnetizations for each wave-number $\boldsymbol{q}$ :

$$
\Delta m_{\boldsymbol{q}_{; \nu}}=\beta \sum_{\nu^{\prime}}\left(\Lambda^{-1}\right) \boldsymbol{q}_{; \nu \nu^{\prime}} H_{\boldsymbol{q} ; \nu^{\prime}}=\sum_{\nu^{\prime}} \chi_{\boldsymbol{q}_{; \nu \nu^{\prime}}} H_{\boldsymbol{q}_{; \nu^{\prime}}}
$$

where $\chi_{\boldsymbol{q} ; \nu \nu^{\prime}}=\beta\left(\Lambda^{-1}\right) \boldsymbol{q}_{; \nu \nu^{\prime}}$ is the wave-number dependent susceptibility. 
Now all we need is to take the inverse of $\Lambda$-matrix with martix elements (3.11). In the present case we can derive its eigenvalues analytically (see appendix B) and these are found to be

$\lambda_{\boldsymbol{q}}^{(1)}=\lambda_{\boldsymbol{q}}^{(2)}=2\left(\Lambda_{\mathrm{diag}}-\Lambda_{\mathrm{off}}\right)$,

$\lambda_{\boldsymbol{q}}^{(3)}=2\left(\Lambda_{\mathrm{diag}}+\Lambda_{\mathrm{off}}\right)-\Lambda_{\mathrm{off}} \sqrt{\sum_{\nu \nu^{\prime}} \cos \left(2 \boldsymbol{q} \cdot\left(\boldsymbol{r}_{\nu}-\boldsymbol{r}_{\nu^{\prime}}\right)\right)}$.

$\lambda_{\boldsymbol{q}}^{(4)}=2\left(\Lambda_{\mathrm{diag}}+\Lambda_{\mathrm{off}}\right)+\Lambda_{\mathrm{off}} \sqrt{\sum_{\nu \nu^{\prime}} \cos \left(2 \boldsymbol{q} \cdot\left(\boldsymbol{r}_{\nu}-\boldsymbol{r}_{\nu^{\prime}}\right)\right)}$.

Note that the eigenvalues $\lambda_{\boldsymbol{q}}^{(1)}$ and $\lambda_{\boldsymbol{q}}^{(2)}$ are degenerated and independent of $\boldsymbol{q}$. Correspondingly the eigenvalues of $\chi \boldsymbol{q}_{; \nu \nu^{\prime}}$ are given as

$$
\chi_{\boldsymbol{q}}^{(\rho)}=\frac{\beta}{\lambda_{\boldsymbol{q}}^{(\rho)}},
$$

and $\chi_{\boldsymbol{q}}^{(1)}$ and $\chi_{\boldsymbol{q}}^{(2)}$ are the largest ones since an inequality

$$
0<\lambda_{\boldsymbol{q}}^{(1)}=\lambda_{\boldsymbol{q}}^{(2)} \leq \lambda_{\boldsymbol{q}}^{(3)} \leq \lambda_{\boldsymbol{q}}^{(4)}
$$

holds for any $\boldsymbol{q}$ and any $T$. This proves the absence of magnetic phase transition in the present approximation because the largest eigenvalue of $\chi \boldsymbol{q}_{; \nu \nu^{\prime}}$ is analytic and never diverges for any temperature $T$. The $\boldsymbol{q}$-dependency of $\chi_{\boldsymbol{q}}^{(\rho)}$ are shown in Fig. 3 .

Once all eigenvalues $\chi_{\boldsymbol{q}}^{(\rho)}$ and the corresponding eigenvectors $u_{\boldsymbol{q} ; \nu}^{(\rho)}$ are known, the $\boldsymbol{q}$-dependent susceptibility matrix $\chi \boldsymbol{q}_{; \nu \nu^{\prime}}$ is expressed as

$$
\chi \boldsymbol{q}_{; \nu \nu^{\prime}}=\sum_{\rho} u_{\boldsymbol{q} ; \nu}^{(\rho)} \chi_{\boldsymbol{q}}^{(\rho)} u_{\boldsymbol{q} ; \nu^{\prime}}^{(\rho)}
$$

The final form of magnetization is thus obtained as follows:

$$
\Delta m_{\boldsymbol{q} ; \nu \alpha}=\sum_{\nu^{\prime} \alpha^{\prime}} \chi{\boldsymbol{q} ; \nu \alpha ; \nu^{\prime} \alpha^{\prime}} H_{\boldsymbol{q} ; \nu^{\prime} \alpha^{\prime}} .
$$

where

$$
\begin{aligned}
& \Delta m_{\boldsymbol{q} ; \nu \alpha}=\Delta m_{\boldsymbol{q} ; \nu} n_{\nu \alpha}, \\
& H_{\boldsymbol{q}_{; \nu}}=\sum_{\alpha} H_{\boldsymbol{q}_{; \nu \alpha}} n_{\nu \alpha}, \\
& \chi_{\boldsymbol{q} ; \nu \alpha ; \nu^{\prime} \alpha^{\prime}}=n_{\nu \alpha} \chi_{\boldsymbol{q} ; \nu \nu^{\prime}} n_{\nu^{\prime} \alpha^{\prime}},
\end{aligned}
$$

and $n_{\nu \alpha}$ is the $\alpha(=x, y, z)$ component of $\boldsymbol{n}_{\nu}$.

\subsection{Neutron Scattering Intensity}

As an application of the present result we evaluate the diffuse magnetic scattering intensity:

$$
\begin{aligned}
I(\boldsymbol{Q}= & \boldsymbol{G}+\boldsymbol{q}) \\
=I_{0} & (f(\boldsymbol{Q}))^{2} \sum_{\nu \alpha ; \nu^{\prime} \alpha^{\prime}}\left(\delta_{\alpha \alpha^{\prime}}-\frac{Q_{\alpha} Q_{\alpha^{\prime}}}{Q^{2}}\right) \\
& \times \chi \boldsymbol{q} ; \nu \alpha ; \nu^{\prime} \alpha^{\prime} \\
& \cos \left(\boldsymbol{G} \cdot\left(\boldsymbol{r}_{\nu}-\boldsymbol{r}_{\nu^{\prime}}\right)\right),
\end{aligned}
$$

where $\boldsymbol{G}, I_{0}$ and $f(\boldsymbol{Q})$ are, respectively, a reciprocal vector, a constant and an atomic form factor. Numerical evaluation of the neutron scattering pattern with $f(\boldsymbol{Q})=1$ is shown in Fig.4. As temperature becomes

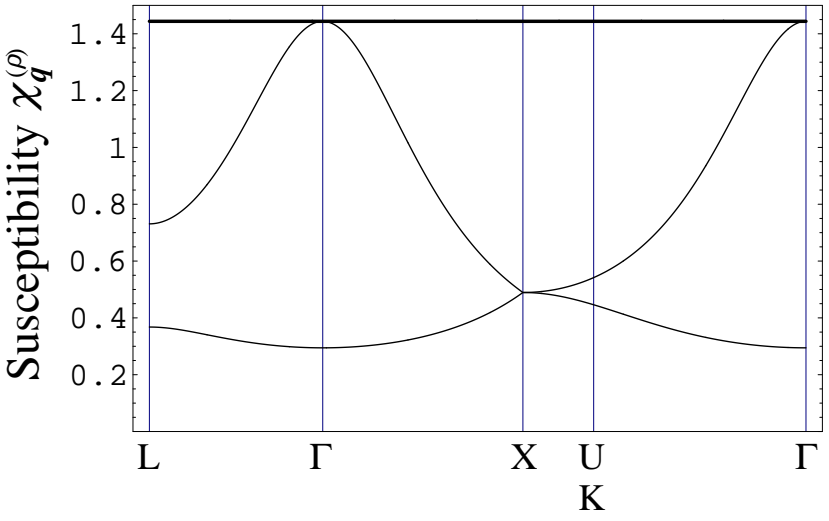

Fig. 3. The $\boldsymbol{q}$-dependency of eigenvalues of $\chi_{\boldsymbol{q} ; \nu \nu^{\prime}}$ in the first Brillouin zone along certain symmetry directions at $k_{\mathrm{B}} T / J=1$.

higher, the calculated pattern becomes vaguer due to the fluctuation. Although almost the same scattering pattern is obtained from the MFA in the paramagnetic phase, the tetrahedral cactus approximation in the CVM shows vaguer pattern than the MFA does at the same temperature. This difference is due to the fluctuation caused by the four spin correlation which the tetrahedral cactus approximation in the CVM can take into account. The scattering pattern agrees qualitatively with the results of Monte Carlo simulation on the basis of the nearest neighbor spin ice model. ${ }^{10)}$

\section{§4. Summary and Discussion}

We have applied the tetrahedral cactus approximation in the CVM to the spin ice system with nearest neighbor interactions. The temperature dependence of the entropy and the specific heat shows qualitatively good agreement with those observed by Monte Carlo simulations and experiments, and the Pauling value is reproduced for the residual entropy. We have obtained the analytic expression of the $\boldsymbol{q}$-dependent magnetic susceptibility and confirmed the absence of magnetic phase transition. Furthermore we have evaluated the neutron scattering pattern, which is also consistent with that obtained from Monte Carlo simulations.

The crucial point which differentiates the present results from those of the MFA is that the tetrahedral cactus approximation can take into account the ice rule in an appropriate manner. It is because that the four-spin correlation between the spins on a tetrahedron plays an important role for the ice rule of spin ice system in low temperatures. To depict this situation let us explain briefly what results in the point approximation of the CVM, which corresponds to the MFA. The approximant $P_{\text {tot }}$ is given by eq.(2.11) and the same procedure as in the previous sections leads to the $\Lambda$-matrix (3.6) with the following parameters replaced by

$$
\Lambda_{\text {diag }}=\frac{1}{2}, \quad \Lambda_{\text {off }}=\frac{J_{\text {eff }}}{3 k_{\mathrm{B}} T} .
$$

The rest of the story, including eqs.(3.13) - (3.15), is also valid for the point approximation and we thus ob- 

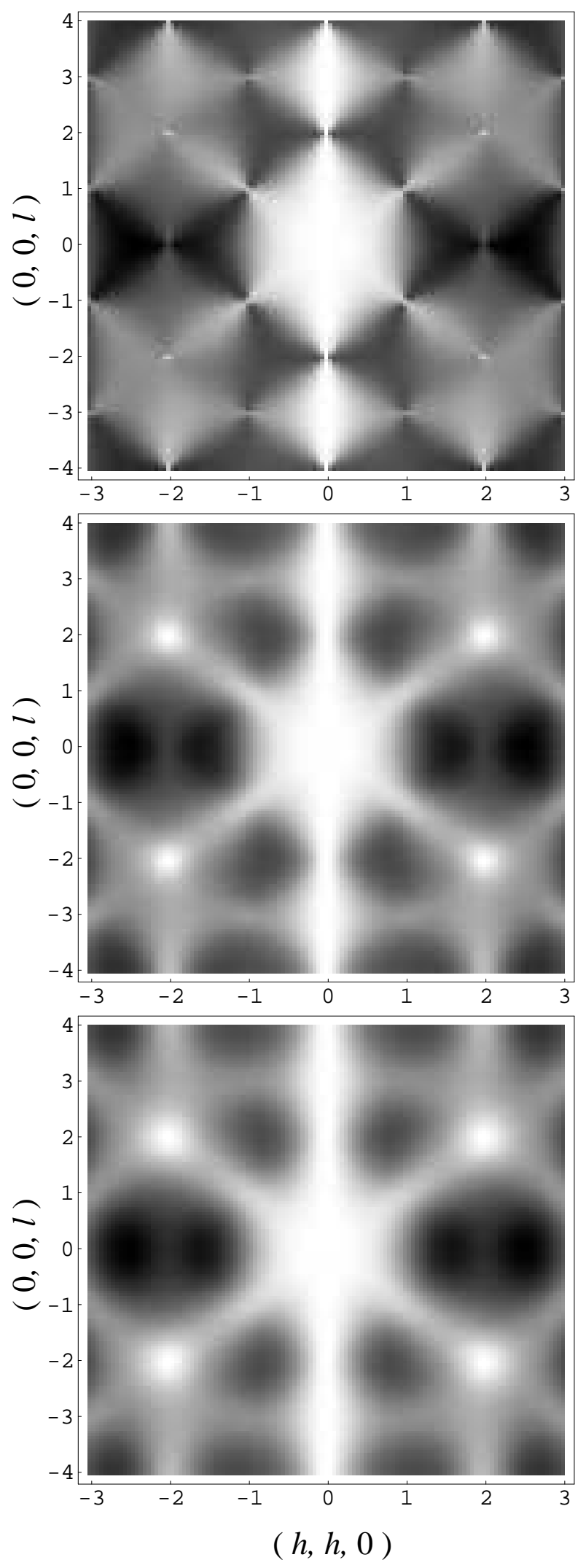

Fig. 4. Neutron scattering pattern in the $(h h l)$ plane of reciprocal space at $k_{\mathrm{B}} T / J=0.1,0.5,1.0$. Black shows the lowest intensity level, white the heighest.



Fig. 5. Inverse of the maximum eigenvalue $\chi_{\boldsymbol{q}}^{(1)}$ versus temperature. The thick and thin lines represent those obtained by the cactus approximation and the usual mean field approximation, respectively.

tain $\chi_{\boldsymbol{q}}^{(\rho)}$. The temperature dependence of the maximum eigenvalue $\chi_{\boldsymbol{q}}^{(1)}$ is shown in Fig.5. In this case it diverges for all $\boldsymbol{q}$ simultaneously at $k_{\mathrm{B}} T_{\mathrm{c}} / J_{\text {eff }}=2 / 3$ since $\chi_{\boldsymbol{q}}^{(1)}=\beta /\left(1-2 \beta J_{\text {eff }} / 3\right)$ in the point approximation. Below $T_{\mathrm{c}}$ the long range order appears and prevents the system from having the expecting residual entropy. Obviously this result is not suitable for the behavior of the present model at low temperatures where the ice rule dominates.

Recently dipolar spin ice systems have been found to show a remarkable aspect. It has been suggested that in the spin ice materials such as $\mathrm{Dy}_{2} \mathrm{Ti}_{2} \mathrm{O}_{7}$ and $\mathrm{Ho}_{2} \mathrm{Ti}_{2} \mathrm{O}_{7}$ the long range dipolar interaction is responsible for spin ice behaviors. It would be then expected that the macroscopic degeneracy of the ground state is removed by the dipolar interaction and the long range order exists at very low temperature. The existence of ordered phase is also indicated by the Monte Carlo simulation by Melko et $a l .{ }^{11)}$ On the other hand, such a phase has not been found in the real spin ice materials $\mathrm{Dy}_{2} \mathrm{Ti}_{2} \mathrm{O}_{7}$ and $\mathrm{Ho}_{2} \mathrm{Ti}_{2} \mathrm{O}_{7} \cdot{ }^{10}$ ) This may happen if with the temperature being decreased the free energy barriers separating the ordered state from quasi-degenerate states grow very high and consequently the relaxation time to the ordered phase becomes very large. In order to elucidate the ordered phase of the dipolar spin ice more in detail, we have to cope with the long range dipolar interactions neglected in the present work. The application of the cactus approximation to the dipolar spin ice is in progress and will be presented elsewhere in the near future.

\section{§5. Acknowledgments}

The authors would like to thank Prof. M. Tokunaga for his encouragements and helpful comments, and Dr K. Matsuhira for introducing us to spin ice systems. 
Press, Ithaca, New York, 1945)

2) M.J.Harris et al., Phys.Rev.Lett. 79,2554(1997).

3) J.F.Nagle, J.Math.Phys. 7,1484(1965).

4) B.C.den Hertog and M.J.P.Gingras, Phys.Rev.Lett 84, 3430 (2000).

5) J.N.Reimers et al., Phys.Rev.B 43,865(1991).

6) H.Kadowaki, Y.Ishii, K.Matsuhira and Y.Hinatsu, condmat/0107278.

7) J.C.Slater, J.Chem.Phys. 9,16(1941).

8) R.Kikuchi, Phys.Rev. 81,988(1951).

9) K.Wada and Y.Ogawa, J.Phys.Soc.Jpn. 67,112(1998).

10) S.T.Bramwel et al., cond-mat/0101114.

11) R.G.Melko et al., cond-mat/0009225.

\section{Appendix A: Derivation of Eq.(3.5)}

First we show the extremum condition (2.20) for the free energy more in detail. For instance, the differentiation with respect to site magnetization $m_{i}$ is written as

$$
\begin{aligned}
\beta \frac{\partial F}{\partial m_{i}}= & \frac{1}{2^{4}} \sum_{\langle j k l\rangle} \operatorname{Tr}\left[\beta \mathcal{H}_{4}^{(i j k l)}\left(\sigma_{i}, \sigma_{j}, \sigma_{k}, \sigma_{l}\right) \sigma_{i}\right] \\
& -\frac{1}{2} \operatorname{Tr}\left[\sigma_{i} \ln P_{1}^{(i)}\left(\sigma_{i}\right)\right] \\
& +\frac{1}{2^{4}} \sum_{\langle j k l\rangle} \operatorname{Tr}\left[\sigma_{i} \ln P_{4}^{(i j k l)}\left(\sigma_{i}, \sigma_{j}, \sigma_{k}, \sigma_{l}\right)\right] .
\end{aligned}
$$

The summation $\sum_{\langle j k l\rangle}$ is taken over two tetrahedra which includes the $i$-th site. By keeping the response of order parameters up to the linear order of external field, eq. $(\mathrm{A} \cdot 1)$ is written as

$$
\begin{aligned}
\beta \frac{\partial F}{\partial m_{i}} \simeq-\beta H_{i}-\Delta m_{i} \\
+\sum_{\langle j k l\rangle} \operatorname{Tr}\left[\frac{\sigma_{i} \Delta P_{4}^{(i j k l)}\left(\sigma_{i}, \sigma_{j}, \sigma_{k}, \sigma_{l}\right)}{2^{4} P_{4}^{0}\left(\sigma_{i}, \sigma_{j}, \sigma_{k}, \sigma_{l}\right)}\right] .
\end{aligned}
$$

Similarly the rests of the eqs.(2.20) are expanded up to the first order as

$$
\begin{gathered}
\beta \frac{\partial F}{\partial m_{a b}} \simeq \operatorname{Tr}\left[\frac{\sigma_{a} \sigma_{b} \Delta P_{4}^{(i j k l)}\left(\sigma_{i}, \sigma_{j}, \sigma_{k}, \sigma_{l}\right)}{2^{4} P_{4}^{0}\left(\sigma_{i}, \sigma_{j}, \sigma_{k}, \sigma_{l}\right)}\right]=0, \\
\beta \frac{\partial F}{\partial m_{a b c}} \simeq \operatorname{Tr}\left[\frac{\sigma_{a} \sigma_{b} \sigma_{c} \Delta P_{4}^{(i j k l)}\left(\sigma_{i}, \sigma_{j}, \sigma_{k}, \sigma_{l}\right)}{2^{4} P_{4}^{0}\left(\sigma_{i}, \sigma_{j}, \sigma_{k}, \sigma_{l}\right)}\right]=0, \\
\beta \frac{\partial F}{\partial m_{i j k l}} \simeq \operatorname{Tr}\left[\frac{\sigma_{i} \sigma_{j} \sigma_{k} \sigma_{l} \Delta P_{4}^{(i j k l)}\left(\sigma_{i}, \sigma_{j}, \sigma_{k}, \sigma_{l}\right)}{2^{4} P_{4}^{0}\left(\sigma_{i}, \sigma_{j}, \sigma_{k}, \sigma_{l}\right)}\right]=0 .
\end{gathered}
$$

where $\Delta$ signifies quantities of linear order to external field. In the paramagnetic phase short range order parameters such as $m_{i j}$ and $m_{i j k l}$ have no linear response because these are even functions of the field. Then eqs. $(\mathrm{A} \cdot 2)$ and $(\mathrm{A} \cdot 4)$ are expressed as:

$$
\begin{gathered}
\beta H_{i}=-\Delta m_{i}+\frac{Z}{2^{8}} \sum_{\langle j k l\rangle}\left(\boldsymbol{M}_{1}^{\mathrm{t}} \Delta \boldsymbol{m}_{1}+\boldsymbol{M}_{3}^{\mathrm{t}} \Delta \boldsymbol{m}_{3}\right) \\
W_{3} \Delta \boldsymbol{m}_{1}+W_{1} \Delta \boldsymbol{m}_{3}=0
\end{gathered}
$$

where a superscript " $\mathrm{t}$ " denotes a transpose of a vector and

$$
\begin{aligned}
& \boldsymbol{m}_{1}=\left(m_{i}, m_{j}, m_{k}, m_{l}\right)^{\mathrm{t}}, \\
& \boldsymbol{m}_{3}=\left(m_{j k l}, m_{k l i}, m_{l i j}, m_{i j k}\right)^{\mathrm{t}} .
\end{aligned}
$$

The 4-column vectors $\boldsymbol{M}_{1}$ and $\boldsymbol{M}_{3}$ are given by

$$
M_{1}^{\mathrm{t}}=(A, B, B, B), \quad M_{3}^{\mathrm{t}}=(C, B, B, B)
$$

and the $4 \times 4$ matrices $W_{1}$ and $W_{3}$ by

$$
\begin{aligned}
& \left(W_{1}\right)_{i j}=A \delta_{i j}+B\left(1-\delta_{i j}\right), \\
& \left(W_{3}\right)_{i j}=C \delta_{i j}+B\left(1-\delta_{i j}\right)
\end{aligned}
$$

with

$$
\begin{aligned}
& A=\operatorname{Tr}\left[\frac{1}{\left.\mathrm{e}^{-\beta \mathcal{H}_{4}^{0}\left(\sigma_{i}, \sigma_{j}, \sigma_{k}, \sigma_{l}\right)}\right]=6 \eta+8+2 \eta^{-3},}\right. \\
& B=\operatorname{Tr}\left[\frac{\sigma_{i} \sigma_{j}}{\left.\mathrm{e}^{-\beta \mathcal{H}_{4}^{0}\left(\sigma_{i}, \sigma_{j}, \sigma_{k}, \sigma_{l}\right)}\right]=-2 \eta+2 \eta^{-3},}\right. \\
& C=\operatorname{Tr}\left[\frac{\sigma_{i} \sigma_{j} \sigma_{k} \sigma_{l}}{\mathrm{e}^{-\beta \mathcal{H}_{4}^{0}\left(\sigma_{i}, \sigma_{j}, \sigma_{k}, \sigma_{l}\right)}}\right]=6 \eta-8+2 \eta^{-3} .
\end{aligned}
$$

By eliminating $\Delta \boldsymbol{m}_{3}$ from eqs.(A.6) and (A.7), the final expression between magnetization and magnetic field is obtained as

$$
\begin{aligned}
\beta H_{i} & =-\Delta m_{i}+\frac{Z}{2^{8}} \sum_{\langle j k l\rangle}\left(\boldsymbol{M}_{1}^{\mathrm{t}}+\boldsymbol{M}_{3}^{\mathrm{t}}\left(W_{1}\right)^{-1} W_{3}\right) \Delta \boldsymbol{m}_{1} \\
& =\sum_{\langle j k l\rangle}\left(\Lambda_{\mathrm{diag}} \Delta m_{i}+\Lambda_{\mathrm{off}}\left(\Delta m_{j}+\Delta m_{k}+\Delta m_{l}\right)\right) .
\end{aligned}
$$

The above equation is easily rewritten in terms of the indexing rule described in $\S 2.1$ to give eq.(3.5).

\section{Appendix B: Derivation of Eq.(3.13)}

We express the $\Lambda$-matrix (3.11) in the form as

$$
\begin{aligned}
\Lambda_{\boldsymbol{q} ; \nu \nu^{\prime}}=2\left(\Lambda_{\mathrm{diag}}-\Lambda_{\mathrm{off}}\right) \delta_{\nu \nu^{\prime}} & \\
+ & 2 \Lambda_{\mathrm{off}} \cos \left(\boldsymbol{q} \cdot\left(\boldsymbol{r}_{\nu}-\boldsymbol{r}_{\nu^{\prime}}\right)\right) .
\end{aligned}
$$

The problem is then reduced to obtaining the eigenvalues of the matrix $\cos \left(\boldsymbol{q} \cdot\left(\boldsymbol{r}_{\nu}-\boldsymbol{r}_{\nu^{\prime}}\right)\right)$ :

$$
\begin{aligned}
\cos & \left(\boldsymbol{q} \cdot\left(\boldsymbol{r}_{\nu}-\boldsymbol{r}_{\nu^{\prime}}\right)\right) \\
& =\cos \left(\boldsymbol{q} \cdot \boldsymbol{r}_{\nu}\right) \cos \left(\boldsymbol{q} \cdot \boldsymbol{r}_{\nu^{\prime}}\right)+\sin \left(\boldsymbol{q} \cdot \boldsymbol{r}_{\nu}\right) \sin \left(\boldsymbol{q} \cdot \boldsymbol{r}_{\nu^{\prime}}\right) \\
& =\left(\boldsymbol{c} \boldsymbol{c}^{\mathrm{t}}+\boldsymbol{s} \boldsymbol{s}^{\mathrm{t}}\right)_{\nu \nu^{\prime}},
\end{aligned}
$$

where

$$
\boldsymbol{c}=\left(\begin{array}{c}
\cos \left(\boldsymbol{q} \cdot \boldsymbol{r}_{1}\right) \\
\cos \left(\boldsymbol{q} \cdot \boldsymbol{r}_{2}\right) \\
\cos \left(\boldsymbol{q} \cdot \boldsymbol{r}_{3}\right) \\
\cos \left(\boldsymbol{q} \cdot \boldsymbol{r}_{4}\right)
\end{array}\right), \quad \boldsymbol{s}=\left(\begin{array}{c}
\sin \left(\boldsymbol{q} \cdot \boldsymbol{r}_{1}\right) \\
\sin \left(\boldsymbol{q} \cdot \boldsymbol{r}_{2}\right) \\
\sin \left(\boldsymbol{q} \cdot \boldsymbol{r}_{3}\right) \\
\sin \left(\boldsymbol{q} \cdot \boldsymbol{r}_{4}\right)
\end{array}\right)
$$

Thus the matrix under consideration is just a sum of projectors. Then it is obvious that the subspace normal to $c$ and $s$ is a two-dimensional eigenspace with the eigenvalue 0 . The rest of the eigenvectors with non-zero eigenvalue can be expressed by a linear combination of $\boldsymbol{c}$ and $s$ as $c_{1} c+c_{2} s$, where $c_{1}$ and $c_{2}$ are chosen so as to 
satisfy

$$
\left(\boldsymbol{c} \boldsymbol{c}^{\mathrm{t}}+\boldsymbol{s s ^ { \mathrm { t } }}\right)\left(c_{1} \boldsymbol{c}+c_{2} s\right)=\kappa\left(c_{1} \boldsymbol{c}+c_{2} s\right),
$$

where $\kappa$ is the eigenvalue. We can rewrite this equation as

$$
\left(\begin{array}{ll}
\boldsymbol{c}^{\mathrm{t}} \boldsymbol{c} & \boldsymbol{s}^{\mathrm{t}} \boldsymbol{c} \\
\boldsymbol{s}^{\mathrm{t}} \boldsymbol{c} & \boldsymbol{s}^{\mathrm{t}} \boldsymbol{s}
\end{array}\right)\left(\begin{array}{l}
c_{1} \\
c_{2}
\end{array}\right)=\kappa\left(\begin{array}{l}
c_{1} \\
c_{2}
\end{array}\right) .
$$

The secular equation is then written as

$$
\operatorname{det}\left(\begin{array}{cc}
\boldsymbol{c}^{\mathrm{t}} \boldsymbol{c}-\kappa & \boldsymbol{s}^{\mathrm{t}} \boldsymbol{c} \\
\boldsymbol{s}^{\mathrm{t}} \boldsymbol{c} & \boldsymbol{s}^{\mathrm{t}} \boldsymbol{s}-\kappa
\end{array}\right)=0,
$$

which can be solved easily to obtain

$$
\kappa=2 \pm \frac{1}{2} \sqrt{\sum_{\nu \nu^{\prime}} \cos \left(2 \boldsymbol{q} \cdot\left(\boldsymbol{r}_{\nu}-\boldsymbol{r}_{\nu^{\prime}}\right)\right)} .
$$

